\title{
Determine the Eigen Function of Schrodinger Equation with Non-Central Potential by Using NU Method
}

\author{
Hamdollah Salehi \\ Department of Physics, Shahid Chamran University, Ahvaz, Iran \\ E-mail: salehi_h@scu.ac.ir \\ Received April 11, 2011; revised June 21, 2011; accepted June 28, 2011
}

\begin{abstract}
So far, Schrodinger equation with central potential has been solved in different methods but solving this equation with non-central potentials is less dealt with. Solving such equations are way more difficult and complicated and a certain and limited number of non-central potentials can be solved. In this paper, we introduce one of the solvable kinds of such potentials and we will use NU method for solving Schrodinger equation and then by using this method we have calculated particular figures of its energy and function.
\end{abstract}

Keywords: Schrodinger Equation (SE), Non-Central Potentials, NU Method, Central Potential

\section{Introduction}

One of the important tasks of quantum mechanic is finding accurate answers of Schrodinger equation with a certain potential. It is obvious that finding exact answers of SE by the usual and traditional methods is impossible, except certain cases such as a system with Qualeny potential or a coordinating oscillator. Thus, it is inevitable to use methods to help us solve this problem. Among the cases where we have to refuse ordinary methods and seek new methods is solving SE with non-central potentials. Such potentials are of high importance in quantum chemistry and nuclear physics. Recently, a lot of studies are being done about such potentials. Accordingly, different methods are used to solve SE with non-central potentials among which we can name symmetrical cloud, SUSY, SIP idea [1,2], route integral [3] and Factorial method [4].

There is also another method known as NU (Nikiforov-Uvarov) which gives a clear instruction for obtaining exact answers of certain states ,Eigen value of energy and the related functions based on Orthogonal polynomials [5]. NU method is based on reducing a second degree differential equation of SE into an equation of hyper geometric type [6-8]. Based on this, in this paper we will try to solve SE with a suitable potential without any limits. Therefore, we consider a potential as follows to meet all our needs in order to solve SE by NU method:

$$
V(r, \theta)=\frac{A}{r}+\frac{B}{r^{2}}+\frac{D \cot ^{2}(\theta)}{r^{2}}
$$

After choosing a suitable change of variable $s=s(r)$, the transformed equation will be as follows:

$$
\Psi_{n}^{\prime \prime}(s)+\frac{\tilde{\tau}(s)}{\sigma(s)} \Psi_{n}^{\prime}(s)+\frac{\tilde{\sigma}(s)}{\sigma^{2}(s)} \Psi_{n}(s)=0
$$

In which $\sigma$ and $\tilde{\sigma}$ are polynomials of maximum second degree and $\tilde{\tau}$ is a polynomial of maximum first degree. By considering wave function $\Psi_{n}(s)$ as:

$$
\Psi_{n}(s)=\varphi_{n}(s) y_{n}(s)
$$

Equation (1) will be reduced to the following equation which is of hyper geometric type [5];

$$
\sigma(s) y_{n}^{\prime \prime}(s)+\tau(s) y_{n}^{\prime}(s)+\lambda y_{n}(s)=0
$$

That in equations:

$$
\begin{gathered}
\sigma(s)=\pi(s) \frac{\phi(s)}{\phi^{\prime}(s)} \\
\tau(s)=\tilde{\tau}(s)+2 \pi(s), \tau^{\prime}<0
\end{gathered}
$$

$\lambda$ is a parameter which is defined as follows[5]:

$$
\lambda=\lambda_{n}=-n \tau^{\prime}(s)-\frac{n(n-1)}{2} \sigma^{\prime \prime}(s), \quad n=0,1,2, \cdots
$$


The polynomial $\tau^{\prime}(s)$ shows that its first derivative must be negative. We should notice that $\lambda$ and $\lambda_{n}$ are obtained from a particular answer of $y(s)=y_{n}(s)$ which is a polynomial of $n$ degree. Furthermore, statement $y_{n}(s)$, wave function of Equation (2), is a function of hyper geometric type which is obtained from the following Rodriguez equation [5].

$$
y_{n}(s)=\frac{\mathrm{B}_{n}}{\rho_{n}} \frac{\mathrm{d}^{n}}{\mathrm{~d} s^{n}}\left(\sigma^{n}(s) \rho(s)\right)
$$

In which $B_{n}$ is the normalization constant and $\rho(s)$ is a weight function which has to meet the following condition $[5,6]$.

$$
\frac{\mathrm{d}}{\mathrm{d} s} \omega(s)=\frac{\tau(s)}{\sigma(s)} \omega(s), \omega(s)=\sigma(s) \rho(s)
$$

Function $\pi(s)$ and parameter $\lambda$ are defined as follow:

$\pi(s)=\frac{\sigma^{\prime}(s)-\tilde{\tau}(s)}{2} \pm \sqrt{\left(\frac{\sigma^{\prime}(s)-\tilde{\tau}(s)}{2}\right)^{2}-\tilde{\sigma}(s)+K \sigma(s)}$

$$
\lambda=K+\pi^{\prime}(s)
$$

Since $\pi(s)$ has to be a polynomial of maximum first degree, the statements under the radical in Equation (9) have to be sorted in the form of a first degree polynomial and this is possible when its determiner, $\Delta=b^{2}-4 a c$, is zero. In this case, an equation is obtained for $\mathrm{K}$ and after solving the equation, the obtained figures for $\mathrm{K}$ are placed in Equation (9) and by comparing with Equations (6) and (10) we will calculate Eigen value of energy.

\section{Schrodinger Equation with Central and Non-Central Potentials}

We consider time-independent SE as follows:

$$
\nabla^{2} \psi(\boldsymbol{r})+\frac{2 m}{\hbar^{2}}(E-V(\boldsymbol{r})) \psi(\boldsymbol{r})=0
$$

Wave function $\Psi_{n}(\boldsymbol{r})$ elaborates certain states and their related energy levels, $E_{n}$, for a particle in a potential field. First, central and non-central potentials

$$
V(\boldsymbol{r})=V(r, \theta)=\frac{A}{r}+\frac{B}{r^{2}}+\frac{D \cot ^{2} \theta}{r^{2}}
$$

are considered in spiracle coordinates and put them in the above equation. ( $2 m=h^{2}=J$ )

$$
\begin{aligned}
& \frac{1}{r^{2}} \frac{\partial}{\partial r}\left(r^{2} \frac{\partial \psi(r)}{\partial r}\right) \\
& +\frac{1}{r^{2}}\left[\frac{1}{\sin ^{2} \theta} \frac{\partial}{\partial \theta}\left(\sin \theta \frac{\partial \psi(r)}{\partial \theta}\right)+\frac{1}{\sin ^{2} \theta} \frac{\partial^{2} \psi(r)}{\partial \varphi^{2}}\right] \\
& +\left[E-\frac{A}{r}-\frac{B}{r^{2}}-\frac{D \cot ^{2} \theta}{r^{2}}\right] \psi(r)=0
\end{aligned}
$$

By considering the whole wave function as:

$$
\Psi(\boldsymbol{r})=\Psi(r, \theta, \phi)=R(r) \mathrm{H}(\theta) \Phi(\phi)
$$

And replacing in Equation (12), we can write the equation separately as follows:

$$
\frac{\mathrm{d}^{2} R(r)}{\mathrm{d} r^{2}}+\frac{2}{r} \frac{\mathrm{d} R(r)}{\mathrm{d} r}+\frac{1}{r^{2}}\left[E r^{2}-A r-B-\gamma\right] R(r)=0
$$

$$
\begin{gathered}
\frac{\mathrm{d}^{2} \mathrm{H}(\theta)}{\mathrm{d} \theta^{2}}+\cot \theta \frac{\mathrm{dH}(\theta)}{\mathrm{d} \theta}+\left[\gamma-\frac{m^{2}}{\sin ^{2} \theta}-D \cot ^{2} \theta\right] \mathrm{H}(\theta)=0 \\
\frac{\mathrm{d}^{2} \Phi(\phi)}{\mathrm{d} \phi^{2}}+m^{2} \Phi(\phi)=0
\end{gathered}
$$

In which $\mathrm{m}^{2}$ and $\gamma=l(l+1)$ are the separation fix amounts. The answer for Equation (16) is as follows [9].

$$
\Phi_{m}(\phi)=\frac{1}{\sqrt{2 \pi}} \mathrm{e}^{ \pm i m \varphi}, \quad m=0, \pm 1, \pm 2, \cdots, \pm l
$$

Equations (14) and (15) are radial and angular equations respectively which we are going to solve by NU method.

\section{Solving Radial Equation and Calculating Eigen Values of Energy by Using NU Method}

For solving radial part of SE, by considering $E=E=-\varepsilon^{2}$ in Equation (14) we will have:

$$
\frac{\mathrm{d}^{2} R(r)}{\mathrm{d} r^{2}}+\frac{2}{r} \frac{\mathrm{d} R(r)}{\mathrm{d} r}+\frac{1}{r^{2}}\left[-\varepsilon^{2} r^{2}-A r-B-\gamma\right] R(r)=0
$$

Now if we compare the above equation with Equation (1), the general form of equation in NU method, we will have:

$$
\tilde{\tau}(r)=2, \sigma(r)=r, \tilde{\sigma}(r)=-\varepsilon^{2} r^{2}-A r-(B+\gamma)
$$

Therefore, according to the definition of $\pi(r)$ in 
Equation (9), we can write $\pi(r)$ as follows:

$$
\pi(r)=-\frac{1}{2} \pm \frac{1}{2} \sqrt{4 \varepsilon^{2} r^{2}}+4(K+A) r+[4(B+\gamma)+1]
$$

From the above equation, considering that under the radical there should be a of a first degree polynomial.

$$
\pi(r)=\left\{\begin{array}{l}
-\frac{1}{2} \pm \frac{1}{2}[2 \varepsilon r+[4(B+\gamma)+1]] \\
-\frac{1}{2} \pm \frac{1}{2}[2 \varepsilon r-[4(B+\gamma)+1]]
\end{array}\right.
$$

We will determine $K$ and we will have:

$$
\begin{aligned}
& K_{1}=-A+\varepsilon[4(B+\lambda)+1]^{1 / 2} \\
& K_{2}=-A+\varepsilon[4(B+\lambda)+1]^{1 / 2}
\end{aligned}
$$

To continue, we choose the suitable amount of $\pi(r)$ which can meet the condition $\tau^{\prime}<0$. So:

$$
\begin{aligned}
& \left\{\begin{array}{l}
\text { (i) } \pi(r)=-\frac{1}{2}-\frac{1}{2}[2 \varepsilon r+[4(B+\gamma)+1]] \\
\text { (ii) } \pi(r)=-\frac{1}{2}-\frac{1}{2}[2 \varepsilon r-[4(B+\gamma)+1]]
\end{array}\right. \\
& K_{1}=-A+\varepsilon[4(B+\lambda)+1]^{1 / 2} \\
& K_{2}=-A+\varepsilon[4(B+\lambda)+1]^{1 / 2}
\end{aligned}
$$

Now, for (i), we can write $\tau(r)$ from Equation (5) as follows:

$$
\tau(r)=-2 \varepsilon r-4(B+\gamma)
$$

And from Equations (6) and (10) we will calculate $\lambda_{n}$ and $\lambda$ respectively

$$
\begin{gathered}
\lambda_{n}=2 n \varepsilon \\
\lambda=\varepsilon\left[[4(B+\gamma)+1]^{\frac{1}{2}}-1\right]-A
\end{gathered}
$$

So:

$$
\lambda=\lambda_{n}, 2 n \varepsilon=-A-\varepsilon\left[(4(B+\lambda)+1)^{1 / 2}+1\right]
$$

And eventually, we can obtain Eigen value of energy for (i) from the above equation:

$$
\varepsilon_{n}=\frac{A}{\left[[4(B+\gamma)+1]^{\frac{1}{2}}-(2 n+1)\right]}
$$

In the same way, for (ii), Equation (22), by repeating the above process we can obtain Eigen value of energy. Therefore, from Equation (5), we write $\tau(r)$ as:

$$
\tau(r)=-2 \varepsilon r+4(B+\gamma)+2
$$

And from Equations (6) and (10) we calculate $\lambda_{n}$ and $\lambda$ :

$$
\begin{gathered}
\lambda_{n}=2 n \varepsilon \\
\lambda=-A-\varepsilon\left[[4(B+\gamma)+1]^{\frac{1}{2}}+1\right] \\
\lambda=\lambda_{n} \quad \therefore 2 n \varepsilon=-A-\varepsilon\left[[4(B+\gamma)+1]^{\frac{1}{2}}+1\right]
\end{gathered}
$$

From the above equations, Eigen value of energy is obtained:

$$
\varepsilon_{n}=\frac{-A}{\left[[4(B+\gamma)+1]^{\frac{1}{2}}+(2 n+1)\right]}
$$

We considered $E=-\varepsilon^{2}$ and from (27) and (32), we have Eigen value of energy as:

$$
E_{n}=\frac{-A^{2}}{\left[[4(B+\gamma)+1]^{\frac{1}{2}} \mp(2 n+1)\right]^{2}}
$$

\section{Calculating Eigen Functions Related to Radial Share of Wave Function}

In order to obtain Eigen radial functions, by using Equation (4) we have:

$$
\frac{\mathrm{d} \varphi}{\mathrm{d} s}=\frac{\pi(s)}{\sigma(s)} \varphi(s), \quad \ln (\phi(s))=\int \frac{\pi(s)}{\sigma(s)} \mathrm{d} s
$$

Thus, for (i) and (ii) in Equation (22) we have:

$$
\begin{aligned}
\pi(r) & =-\frac{1}{2}-\frac{1}{2}[2 \varepsilon r \pm[4(B+\gamma)+1]] \\
& =-\frac{1}{2}-\frac{1}{2}[2 \varepsilon r \pm \delta]
\end{aligned}
$$

in which $\delta=4[B+\gamma]+1$ and considering $\sigma(r)=r$ We will have:

$$
\left\{\begin{array}{l}
\text { i) } \phi(r)=r^{-\frac{(\delta+1)}{2}} \mathrm{e}^{-\varepsilon r} \\
\text { ii) } \phi(r)=r^{\frac{(\delta-1)}{2}} \mathrm{e}^{-\varepsilon r}
\end{array}\right.
$$

On the other hand, from Equation (8) we have:

$$
\frac{\mathrm{d} w(s)}{\mathrm{d} s}=\frac{t(s)}{s(S)} w(s) \rightarrow \ln (w(s))=\int \frac{t(s)}{s(S)} \mathrm{d} s
$$

From Equation (37), as we have $\tau(r)=-2 \varepsilon r-\delta^{\prime}$ in which $\delta^{\prime}=\delta-1$, we will have:

$$
\omega(r)=r^{-\delta^{\prime}} \mathrm{e}^{-2 \varepsilon r}
$$


So we can write the weight function $\rho(r)=\frac{\omega(r)}{\sigma(r)}$ as follows:

$$
\rho(r)=r^{-\delta} \mathrm{e}^{-2 \varepsilon r}
$$

And to continue, from Equation (7), we will write $y_{n}(r)$ as follows:

$$
y_{n}(r)=\frac{B_{n}}{r^{-\delta} \mathrm{e}^{-2 \varepsilon r}} \frac{\mathrm{d}^{n}}{\mathrm{~d} r^{n}}\left(r^{n-\delta} \mathrm{e}^{-2 \varepsilon r}\right)
$$

From Rodriguez sequence polynomial, we will have Laguerres dependent functions as [9]:

$$
L_{n}^{k}(x)=\frac{\mathrm{e}^{x} x^{-k}}{n !} \frac{\mathrm{d}^{n}}{\mathrm{~d} x^{n}} \mathrm{e}\left(\mathrm{e}^{-x} x^{n+k}\right)
$$

By comparing the two functions, (39) and (40) and by considering $k=-\delta$ and $2 \varepsilon r=x$ we will have:

$$
y_{n}(r)=B_{n} n !(-1)^{n} L_{n}^{-\delta}(2 \varepsilon r)
$$

In the same way for (ii) in which $\tau(r)=-2 \varepsilon r+\delta+1$ from Equation (37) we will have:

$$
\begin{gathered}
\omega(r)=r^{(\delta+1)} \mathrm{e}^{-2 \varepsilon r} \\
\rho(r)=\frac{\omega(r)}{\sigma(r)}=r^{\delta} \mathrm{e}^{-2 \varepsilon r}
\end{gathered}
$$

And finally, from Equation (7), we can write $y_{n}(r)$ as follows:

$$
y_{n}(r)=\frac{B n}{r^{\delta} \mathrm{e}^{-2 \varepsilon r}} \frac{\mathrm{d}^{n}}{\mathrm{~d} r^{n}}\left(r^{n+\delta} \mathrm{e}^{-2 \varepsilon r}\right)
$$

By comparing Equations (41) and (45) and considering $k=\delta$ and $x=2 \varepsilon r$ we will have:

$$
\text { (ii) } y_{n}(r)=B_{n} n !(-1)^{n} L_{n}^{\delta}(2 \varepsilon r)
$$

Therefore, from Equations (2), (35), (36), (42), (46) the whole radial wave function can be written as:

$$
\left\{\begin{array}{l}
\text { (i) } R(r)=B_{n}(-1)^{n} n ! r^{-\frac{(\delta+1)}{2}} \mathrm{e}^{-\varepsilon r} L_{n}^{-\delta}(2 \varepsilon r) \\
\text { (ii) } R(r)=B_{n}(-1)^{n} n ! r^{\frac{(\delta-1)}{2}} \mathrm{e}^{-\varepsilon r} L_{n}^{\delta}(2 \varepsilon r)
\end{array}\right.
$$

Normalization coefficient is determined by $\int_{0}^{\infty} r^{2}[R(r)]^{2} \mathrm{~d} r=1$ by taking orthogonal condition of associated Laguerre polynomials and we will have:

$$
\left\{\begin{array}{l}
\text { i) } B_{n}=\sqrt{\frac{(2 \varepsilon r)^{-\delta+2}}{n !(n-\delta) !(2 n-\delta+1)}} \\
\text { ii) } B_{n}=\sqrt{\frac{(2 \varepsilon r)^{\delta+2}}{n !(n+\delta) !(2 n+\delta+1)}}
\end{array}\right.
$$

\section{Solving Angular Equation and Calculating Eigen Values}

With a variable change as $x=\cos \theta$ we will have Equation (15) as the following transformation:

$$
\begin{aligned}
& \frac{\mathrm{d}^{2} \mathrm{H}(x)}{\mathrm{d} x^{2}}-\frac{2 x}{1-x^{2}} \frac{\mathrm{dH}(x)}{\mathrm{d} x} \\
& +\frac{1}{\left(1-x^{2}\right)^{2}}\left[\gamma\left(1-x^{2}\right)-m^{2}-D x^{2}\right] \mathrm{H}(x)=0
\end{aligned}
$$

By comparing Equations (49) with (1) we will have:

$$
\begin{aligned}
& \tilde{\tau}(x)=-2 x, \sigma(x)=\left(1-x^{2}\right) \\
& \tilde{\sigma}(x)=-(\gamma+D) x^{2}+\left(\gamma-m^{2}\right)
\end{aligned}
$$

By using (9), we write $\pi(x)$ as:

$$
\pi(x)= \pm \sqrt{(\gamma+D-K) x^{2}+\left(m^{2}-\gamma+K\right)}
$$

We determine $K$ on condition that there is a first degree polynomial under the radical:

$$
\begin{gathered}
\pi(x)=\left\{\begin{array}{l} 
\pm \sqrt{m^{2}+D} \\
\pm\left[\sqrt{m^{2}+D}\right] x
\end{array}\right. \\
K_{1}=\gamma+D \\
K_{2}=\gamma-m^{2}
\end{gathered}
$$

And for $\tau^{\prime}<0$ we have the right choice as:

$$
\begin{gathered}
\pi(x)=-\left(m^{2}+D\right)^{1 / 2} x \\
K_{2}=\gamma-m^{2}
\end{gathered}
$$

Also, from Equation (5), we can obtain $\tau(x)$ Then we will have:

$$
\tau(x)=-2\left(1+\left(D+m^{2}\right)^{1 / 2}\right) x
$$

According to Equations (6) and (10), we will calculate $\lambda_{n}$ and $\lambda$ respectively:

$$
\begin{aligned}
& \lambda_{n}=2 n\left(1+\left(m^{2}+D\right)^{\frac{1}{2}}\right)-n(n-1) \\
& \lambda=\left(\gamma-m^{2}\right)-\left(D+m^{2}\right)^{1 / 2}, \lambda=\lambda_{n}
\end{aligned}
$$

Finally we will obtain $\gamma$ from the above equation:

$$
\gamma=n(3-n)+(2 n+1)\left(D+m^{2}\right)^{1 / 2}+m^{2}
$$




\section{Calculation Eigen Functions of Angular Equation}

To find Eigen function related to angular equation, by using Equation (4) we will have:

$$
\ln (\phi(s))=\int \frac{\pi(s)}{\sigma(s)} \mathrm{d} s
$$

Thus, for $\pi(x)=-\alpha x$ in which $\alpha=\left(D+m^{2}\right)^{1 / 2}$ and considering that $\sigma(x)=\left(1-x^{2}\right)$ we will have:

$$
\varphi(x)=\left(1-x^{2}\right)^{\alpha / 2}
$$

On the other hand, from Equation (8) we have:

$$
\ln (\omega(s))=\int \frac{\tau(s)}{\sigma(s)} \mathrm{d} s
$$

As $\tau(x)=-2(1+\alpha) x$, so:

$$
\rho(x)=\left(1-x^{2}\right)^{\alpha}
$$

Therefore, we can write the weight function $\rho(r)=\frac{\omega(r)}{\sigma(r)}$ as follows:

$$
\rho(x)=\left(1-x^{2}\right)^{\alpha}
$$

And eventually, from Equation (7), we write $y_{n}(x)$ as:

$$
y_{n}(x)=\frac{B_{n}}{\left(1-x^{2}\right)^{\alpha}} \frac{\mathrm{d}^{n}}{\mathrm{~d} x^{n}}\left[\left(1-x^{2}\right)^{n+\alpha}\right]
$$

From Rodriguez sequence, we will have Jacobi's polynomials as $[10,11]$ :

$$
\begin{aligned}
P_{n}^{\left(\alpha^{\prime}, \beta^{\prime}\right)}(z) & =\frac{1}{2^{n} n !}(z-1)^{\alpha^{\prime}}(z+1)^{-\beta^{\prime}} \\
& \cdot \frac{\mathrm{d}^{n}}{\mathrm{~d} z^{n}}\left[(z-1)^{\left(n+\alpha^{\prime}\right)}(z+1)^{\left(n+\beta^{\prime}\right)}\right]
\end{aligned}
$$

We will rewrite Equation (64) as:

$$
\begin{aligned}
y_{n}(x) & =\left[B_{n} 2^{n} n !(-1)^{n}(x-1)^{-2 \alpha}\right] \frac{1}{2^{n} n !}(x-1)^{\alpha} \\
& \cdot(x+1)^{-\alpha} \frac{\mathrm{d}^{n}}{\mathrm{~d} x^{n}}\left[(x-1)^{n+\alpha}(x+1)^{n+\alpha}\right]
\end{aligned}
$$

Now, by comparing Equations (65) and (64) and considering $\alpha^{\prime}, \beta^{\prime}=\alpha$ we will have:

$$
y_{n}(x)=\left[B_{n} 2^{n} n !(-1)^{n}\right](x-1)^{-2 \alpha} P_{n}^{(\alpha, \alpha)}(x)
$$

And finally angular wave function will be obtained by using Equations (2), (60) and (65):

$$
\begin{aligned}
\mathrm{H}_{n}(x) & =\phi_{n}(x) y_{n}(x) \\
& =\left[B_{n} 2^{n} n !(-1)^{n}\right](x-1)^{-2 \alpha}\left(1-x^{2}\right)^{\frac{\alpha}{2}} P_{n}^{(\alpha, \alpha)}(x)
\end{aligned}
$$

This way, we calculated Eigen of functions and Eigen value of energy. According to this wave function, we can determine static characteristics of this system. We can also use this method for solving SE with other non-central potentials.

\section{Conclusions}

In this paper we showed that Eigen value of energy and its Eigen dependent functions can be obtained for a system under a certain non-central potential by using the NU method. Of course, we should note that this method, too, like previous methods for solving SE, does not function with any non-central potential and we can get a suitable result out of this method only with certain types of potentials which meet the requirements of the method. And in this paper, by considering all the requirements, we have introduced the potential in mind and gotten the results mentioned in the article.

\section{References}

[1] B. Gonu and I. Zorba, "Supersymmeric Solutions of Noncentral Potentials,” Physics Letters A, Vol. 269, No. 2-3, 2000, pp. 83-88. doi:10.1016/S0375-9601(00)00252-8

[2] R. Dutt, A. Gangopadhyaya and U. P Sukhatme, "Noncentral Potentials and Spherical Harmonics Using Supersymmetry and Shape Invariance," American Journal of Physics, Vol. 65, 1997, pp. 400-403.

[3] Y. Aharonov and D. Bohn, "On the Measurement of Velocity of Relaivistic Particles,” Il Nuovo Cimento (19551965), Vol. 5, No. 3, 1957, pp. 429-439.

[4] B. M. Mandel, "Path Integral Solution of Noncentral Potential,” International Journal of Modern Physics A, Vol. 15, No. 9, 2000, pp. 1225-1238.

[5] A. F. Nikiforov and V. B. Uvarov, "Special Functions of Mathematical Physics Birkhauser,” Basel, 1988.

[6] H. F. Jones and R. J. Rivers, "Which Green Functions does the Path Integral for Quasi-Hermitian Hamiltonians Represent?” Physics Letters A, Vol. 373, No. 37, 2009, pp. 3304-3308. doi:10.1016/j.physleta.2009.07.034

[7] S. Sakoda, "Exactncess in the Path Integral of Coulomb Potential in One Space Dimension," Modern Physics Letters A, Vol. 23, No. 36, 2008, pp. 3057-3076. doi:10.1142/S0217732308028491

[8] F. Cooper, J. Ginocchio and A. Khare, "Relationship between Suprsymmetry and Solvable Potentials," Physi- 
cal Review D, Vol. 36, No. 8, 1987, pp. 2458-2473. doi:10.1103/PhysRevD.36.2458

[9] R. Dutt, A. Khare and U. P. Sukhatme, "Is the Lowest Order Supersymmetric WKB Approximation Exact for All Shape Invariant Potentials," American Journal of Physics, Vol. 56, No. 2, 1988, pp. 163-168. doi:10.1119/1.15697
[10] H. Katsura, H. Aoki and J. Math, "Exact Supersymmetry in the Relativistic Hydrogen Atom in General Dimension Superchange and Gerelized Johnson-Lippman Operator,” Physics, Vol. 47, 2006, pp. 032301.

[11] G. Arfken, "Mathematical Methods for Physics," 3rd Edition, Academic Press, Waltham, 1985. 